\title{
INSTANTANEOUS SHRINKING IN NONLINEAR DIFFUSION-CONVECTION
}

\author{
B. H. GILDING AND R. KERSNER \\ (Communicated by Barbara L. Keyfitz)
}

\begin{abstract}
The Cauchy problem for a nonlinear diffusion-convection equation is studied. The equation may be classified as being of degenerate parabolic type with one spatial derivative and a time derivative. It is shown that under certain conditions solutions of the initial-value problem exhibit instantaneous shrinking. This is to say, at any positive time the spatial support of the solution is bounded above, although the support of the initial data function is not. This is a phenomenon which is normally only associated with nonlinear diffusion with strong absorption. In conjunction, a previously unreported phenomenon is revealed. It is shown that for a certain class of initial data functions there is a critical positive time such that the support of the solution is unbounded above at any earlier time, whilst the opposite is the case at any later time.
\end{abstract}

In this article we shall consider the Cauchy problem

$$
\begin{gathered}
u_{t}=\left(u^{m}\right)_{x x}+\left(u^{n}\right)_{x} \quad \text { in } \mathbf{R} \times \mathbf{R}^{+}, \\
u(x, 0)=u_{0}(x) \quad \text { for } x \in \mathbf{R},
\end{gathered}
$$

where $m$ and $n$ are positive real constants and $u_{0}$ is a given bounded continuous nonnegative function.

Because, in general, problem (1), (2) does not admit a classical solution [4, $8,10,12]$, it is necessary to introduce the notion of a generalized solution. We shall say that a function $u(x, t)$ is a generalized supersolution of equation (1) in a domain

$$
D=\left(\eta_{1}, \eta_{2}\right) \times(0, T]
$$

with

$$
-\infty \leq \eta_{1}<\eta_{2} \leq \infty \text { and } 0<T<\infty
$$

Received by the editors June 15,1988 and, in revised form, July 6, 1989.

1980 Mathematics Subject Classification (1985 Revision). Primary 35K55, 35K65, 35B99.

Key words and phrases. Nonlinear degenerate parabolic equation, diffusion, convection, qualitative behavior.

Research was supported in part through Research Fund 1386261 of the Hungarian Academy of Sciences. 
if it is defined, real, nonnegative, bounded, and continuous in $\bar{D}$ and satisfies the integral inequality

$$
\begin{aligned}
\int_{t_{1}}^{t_{2}} \int_{x_{1}}^{x_{2}}\left\{u \phi_{t}+u^{m} \phi_{x x}-u^{n} \phi_{x}\right\} d x d t \\
\leq \int_{x_{1}}^{x_{2}}\left\{u\left(x, t_{2}\right) \phi\left(x, t_{2}\right)-u\left(x, t_{1}\right) \phi\left(x, t_{1}\right)\right\} d x \\
\quad+\int_{t_{1}}^{t_{2}}\left\{u^{m}\left(x_{2}, t\right) \phi_{x}\left(x_{2}, t\right)-u^{m}\left(x_{1}, t\right) \phi_{x}\left(x_{1}, t\right)\right\} d t
\end{aligned}
$$

for all bounded rectangles $R=\left(x_{1}, x_{2}\right) \times\left(t_{1}, t_{2}\right] \subseteq D$ and nonnegative functions $\phi \in C^{2,1}(\bar{R})$ such that $\phi\left(x_{1}, t\right)=\phi\left(x_{2}, t\right)=0$ for all $t \in\left[t_{1}, t_{2}\right]$. Similarly, we shall say that a function is a generalized subsolution of equation (1) in $D$ if it satisfies the previous criteria with the inequality sign in (5) reversed. We define a generalized solution of problem (1), (2) as a function $u(x, t)$ which is a generalized supersolution and a generalized subsolution of (1) in all domains of the form (3), (4) and which satisfies (2).

With the above-mentioned notion of a generalized solution, problem (1), (2) has been shown to be uniquely solvable. Furthermore, for any generalized solution of problem (1), (2), $u(x, t)$, the following comparison principle has been proven. If $v(x, t)$ denotes a generalized supersolution of equation (1) in a domain $D$ of the form (3), (4) such that $v(x, t) \geq u(x, t)$ for all $(x, t) \in \bar{D} \backslash D$ then $v(x, t) \geq u(x, t)$ for all $(x, t) \in \bar{D}$. Correspondingly, if $v(x, t)$ denotes a generalized subsolution of equation (1) in a domain $D$ of the type (3), (4) such that $v(x, t) \leq u(x, t)$ for all $(x, t) \in \bar{D} \backslash D$ then $v(x, t) \leq u(x, t)$ for all $(x, t) \in \bar{D}[4,8]$.

Suppose now that $u_{0}$ is not identically zero, and let $u(x, t)$ denote the unique generalized solution of problem (1), (2). Define

$$
\zeta^{-}(t)=\inf \{x \in \mathbf{R}: u(x, t)>0\}
$$

and

$$
\zeta^{+}(t)=\sup \{x \in \mathbf{R}: u(x, t)>0\}
$$

for all $t \geq 0$, i.e. let $\zeta^{ \pm}$denote the limits of the support of the solution as a function of $t$. When they are finite these functions are interfaces or free boundaries entering the solution of problem (1), (2), and it is at points on these interfaces that the generalized solution $u(x, t)$ may fail to be a classical solution $[4,7,8,12]$.

As $u_{0}$ is not identically zero

$$
-\infty \leq \zeta^{-}(t)<\zeta^{+}(t) \leq \infty \quad \text { for all } t \geq 0
$$

[7]. Moreover, the following can be stated when $n \geq 1$ [7, 10, 12]:

(i) If $m \leq 1$ or $\pm \zeta^{ \pm}(0)=\infty$ then $\pm \zeta^{ \pm}(t)=\infty$ for all $t>0$.

(ii) If $m>1$ and $\pm \zeta^{ \pm}(0)<\infty$ then $\pm \zeta^{ \pm}(t)<\infty$ for all $t>0$. 
In this respect, equation (1) resembles the well-known porous media equation which may be obtained by deleting the term $\left(u^{n}\right)_{x}$ in (1), and which, as a prototype for nonlinear diffusion equations, has been the subject of considerable study in recent years $[1,2,12]$. In contrast, when $n<1$, equation (1) gives rise to behavior which is quite different from that exhibited by solutions of the porous media equation. The following is known to be the case when $n<1$ $[3,7]$ :

(i) If $m \leq n$, then irrespective of the value of $\zeta^{ \pm}(0), \pm \zeta^{+}(t)=\infty$ for all $t>0$.

(ii) If $m>n$, then irrespective of the value of $\zeta^{-}(0),-\zeta^{-}(t)=\infty$ for all $t>0$.

(iii) If $m>n$ and $\zeta^{+}(0)<\infty$ then $\zeta^{+}(t)<\infty$ for all $t>0$.

In this article we shall shed light on the remaining possibility when $n<1$, namely $m>n$ and $\zeta^{+}(0)=\infty$, by proving the following theorem.

Theorem 1. Suppose that $n<1, m>n$ and

$$
u_{0}(x) \sim C x^{-1 /(1-n)} \quad \text { as } x \rightarrow \infty
$$

for some constant $C>0$. Then

$$
\zeta^{+}(t)=\infty \quad \text { for all } t \in\left(0, C^{1-n} / n\right)
$$

and

$$
\zeta^{+}(t)<\infty \quad \text { for all } t \in\left(C^{1-n} / n, \infty\right) .
$$

In view of the comparison principle for solutions of problem (1), (2), this theorem infers that if

$$
u_{0}(x)=o\left(x^{-1 /(1-n)}\right) \quad \text { as } x \rightarrow \infty
$$

and $u_{0}$ is not identically zero for large $x$, then there is instantaneous shrinking of the support of the solution of problem (1), (2). This is to say, despite the fact that $\zeta^{+}(0)=\infty, \zeta^{+}(t)<\infty$ for any $t>0$.

This remarkable behavior has been proved for the Cauchy problem for the equation

$$
u_{t}=(a(u))_{x x}+(b(u))_{x}-c(u),
$$

where $a, b \in C^{1}([0, \infty))$ and $C \in C([0, \infty))$ satisfy

$$
\begin{gathered}
a(0)=0, \quad a^{\prime}(s)>0 \quad \text { for all } s>0, \\
b^{\prime}(s) / a^{\prime}(s) \text { is bounded on compact subsets of }[0, \infty), \\
c(0)=0, \quad c(s)>0 \quad \text { for all } s>0,
\end{gathered}
$$

and

$$
a^{\prime}(s)\{a(s) c(s)\}^{-1 / 2},\{c(s)\}^{-1} \in L^{1}(0,1),
$$


together with certain other conditions, by Evans and Knerr [5]. For the particular case

$$
u_{t}=\left(u^{m}\right)_{x x}+\left(u^{n}\right)_{x}-u^{p},
$$

with $m, n$ and $p$ real constants, these conditions are equivalent to

$$
\begin{aligned}
& m \geq 1, \\
& n=0 \quad \text { or } \quad n \geq m,
\end{aligned}
$$

and

$$
0<p<1 .
$$

Instantaneous shrinking has also been established for the equation

$$
u_{t}=u_{x x}-\left(1+x^{2}\right)^{-1 / 2} c(u)
$$

where

$$
c(u)=u^{p} \quad \text { with } 0<p<1,
$$

by Kalashnikov [11]. However, to the best of the authors' knowledge, this is the first time that this phenomenon has been shown to occur for an equation of the form (8) (or (10)) with the term $c$ absent.

Equations of the general type (8) arise in a number of physical situations. In these contexts, $t$ denotes time and $x$ a spatial coordinate. Furthermore, the term $(a(u))_{x x}$ is commonly referred to as the diffusion term, $(b(u))_{x}$ as the convection term, and $c(u)$ as the absorption or sink term. Apparently, for equation (1) with $n<1$ and $n<m$, the convection mechanism is sufficiently powerful in comparison to the diffusive mechanism that in some way the equation emulates nonlinear diffusion with strong absorption.

Theorem 1 though brings to light a previously unreported phenomenon which is even more remarkable than instantaneous shrinking alone. One could speak of deferred instantaneous shrinking. When (7) holds, there is a critical time $\tau^{*}=C^{1-n} / n, 0<\tau^{*}<\infty$, such that the support of the solution is unbounded above for any $t<\tau^{*}$, whilst the opposite is the case for any $t>\tau^{*}$. What actually happens at the critical time $\tau^{*}$ is a riddle.

To prove Theorem 1 we shall utilize the following result which is of some inherent interest.

Theorem 2. Suppose that $n<1, m>n$ and

$$
u_{0}(x) \leq C x^{-\gamma} \quad \text { for all } x \geq x_{0}
$$

for some constants $C>0$,

$$
\gamma>1 /(1-n)
$$

and $x_{0}>0$. Set

$$
\sigma=1 /\{(1-n) \gamma-1\}
$$

and

$$
\mu=(n \omega \gamma)^{-\sigma} \quad \text { where } \omega=\min \{m, 1\}-n \text {. }
$$


Then, given any $\varepsilon>0$ there exists a $T>0$ such that

$$
\zeta^{+}(t) \leq\left(\mu C^{(1-n) \sigma}+\varepsilon\right) t^{-\sigma} \quad \text { for all } t \in(0, T] .
$$

Theorem 2 provides a lower estimate of the speed of shrinking under conditions appropriate to instantaneous shrinking.

The remainder of this article is devoted to the proof of Theorems 1 and 2 . First, we shall prove Theorem 1 assuming that Theorem 2 is true. Thereafter, we prove Theorem 2 .

We refer the interested reader to $[7,9,12,15]$ for additional information on the qualitative behavior of solutions of problem (1), (2) and their associated interfaces $\zeta^{ \pm}$, and to $[1,2,12]$ for surveys of work on these and related topics. Data on diverse characteristics of nonnegative solutions of equation (9) such as the occurrence of similar interfaces in generalized solutions of this equation, the localization and extinction of solutions, and on the role in which the exponents in the diffusion term, the convection term and the absorption term in (9) play in determining these characteristics may be found in $[6,10,13-15]$.

\section{THE PROOF OF THEOREM 1}

To prove the theorem we shall use the following two lemmas.

Lemma 1. Suppose that

$$
u_{0}(x) \geq C x^{-1 /(1-n)} \quad \text { for all } x \geq x_{0}
$$

for some $C>0$ and $x_{0}>0$. Then $\zeta^{+}(t)=\infty$ for all $t \in\left[0, C^{1-n} / n\right)$.

Proof. Let $P_{0}=\left\{x \in \mathbf{R}: u_{0}(x)>0\right\}$. In view of the comparison principle for generalized solutions of problem (1), (2), without loss of generality we may suppose that $P_{0}$ is connected. Set $T=C^{1-n} / n$.

Recalling (6), $\sup \{u(x, T): x \in \mathbf{R}\}>0$. Hence, utilizing an argument in [7] there exists a function $\xi \in C([0, T])$ such that $u(\xi(t), t)>0$ for all $t \in$ $[0, T]$. Furthermore, since $P_{0}$ is connected, $u_{0}(x)>0$ for all $x \in\left[\xi(0), x_{0}\right]$. Consequently, if we define

$$
\Omega=\{(x, t): \xi(t)<x<\infty, 0<t \leq T\}
$$

and

$$
v(x, t)=C(x+\rho)^{-1 /(1-n)}(1-t / T)^{1 /(1-n)},
$$

we can pick a $\rho$ sufficiently large that $x+\rho>0$ and

$$
v(x, t) \leq u(x, t) \quad \text { for all }(x, t) \in \bar{\Omega} \backslash \Omega .
$$

Letting $N$ denote the nonlinear parabolic differential operator

$$
N(z)=\left(z^{m}\right)_{x x}+\left(z^{n}\right)_{x}-z_{t},
$$

by direct computation

$$
N(v)=m(m+1-n)(1-n)^{-2}(x+\rho)^{-2} v^{m} \geq 0 \quad \text { in } \Omega .
$$


So $v$ satisfies (11) and is a classical subsolution of equation (1) in $\Omega$.

From the last remark we would like to be able to conclude that

$$
v(x, t) \leq u(x, t) \quad \text { for all }(x, t) \in \bar{\Omega} .
$$

Unfortunately the quoted comparison principle cannot deliver this conclusion since the domain $\Omega$ is not of the form (3), (4). However [8], $u$ can be constructed on the domain $(-\infty, \infty) \times(0, T]$ as the pointwise limit of a decreasing sequence of positive classical solutions of equation (1), $\left\{u_{k}\right\}_{k=1}^{\infty}$, each one of which is defined on the corresponding member of an expanding sequence of bounded domains $\left\{\bar{Q}_{k}\right\}_{k=1}^{\infty}$, where

$$
\begin{aligned}
& Q_{k}=(-k, k) \times(0, T], \\
& u_{k}(x, 0) \geq u_{0}(x) \quad \text { for all } x \in[-k, k], \\
& u_{k}( \pm k, t) \geq M \quad \text { for all } t \in[0, T],
\end{aligned}
$$

and

$$
M=\sup \{u(x, t):-\infty<x<\infty, 0 \leq t \leq T\} .
$$

Thus, by applying the classical maximum principle to compare $v$ with each solution $u_{k}$ in $\Omega \cap Q_{k}$ and then taking limits, we can still draw the conclusion (13).

From (13) we deduce that $\zeta^{+}(t)=\infty$ for all $t \in[0, T)$.

Lemma 2. Suppose that

$$
u_{0}(x) \leq C x^{-1 /(1-n)} \quad \text { for all } x \geq x_{0}
$$

for some $C>0$ and $x_{0}>0$. Then given any $T>C^{1-n} / n$ and $\alpha>0$ there exists $a K>0$ and an $x_{1} \geq x_{0}$ such that

$$
u(x, T) \leq K x^{-(1+\alpha) /(1-n)} \quad \text { for all } x \geq x_{1} .
$$

Proof. Fix $T>C^{1-n} / n$ and $\alpha>0$. Let

$$
M=\sup \left\{u_{0}(x): x \in \mathbf{R}\right\}
$$

and

$$
\gamma=1 /(1-n),
$$

and for $x_{1} \geq x_{0}$ consider the function

$$
v(x, t)=C x^{-\gamma}\left(1-t / T+M^{1-n} C^{n-1} x_{1}^{1+\alpha} x^{-\alpha}\right)^{\gamma} .
$$

Set

$$
\Omega=\left\{(x, t) \in\left(x_{1}, \infty\right) \times(0, T]: v(x, t)<M\right\} .
$$

We assert that we can choose $x_{1}$ sufficiently large that $v$ is a classical supersolution of equation (1) in $\Omega$. 
Assuming for the moment that $v$ is indeed a classical supersolution of equation (1) in $\Omega$, it can be verified that the function $w$ defined in the closure of the domain

$$
D=(-\infty, \infty) \times(0, T]
$$

by

$$
w(x, t)= \begin{cases}v(x, t) & \text { for }(x, t) \in \bar{\Omega} \\ M & \text { for }(x, t) \in \bar{D} \backslash \bar{\Omega}\end{cases}
$$

is a generalized supersolution of equation (1) in this new domain. Furthermore, if $x^{*}=\sup \{x \in \mathbf{R}: w(x, 0)=M\}$, we have

$$
w(x, 0)=M \geq u_{0}(x) \quad \text { for all } x \leq x^{*}
$$

and

$$
w(x, 0)=v(x, 0)>C x^{-\gamma} \geq u_{0}(x) \quad \text { for all } x \geq x^{*} .
$$

Hence, by the comparison principle for generalized solutions of problem (1), (2),

$$
w(x, T) \geq u(x, T) \quad \text { for all } x \in \mathbf{R} .
$$

However, since

$$
w(x, T)=v(x, T)=M\left(x_{1} / x\right)^{\gamma(1+\alpha)} \quad \text { for all } x \geq x_{1},
$$

this proves the lemma.

To complete the proof of the lemma it therefore remains to demonstrate that we can choose an $x_{1}$ sufficiently large that $v$ is a classical supersolution of equation (1) in $\Omega$. With $N$ given by (12), we calculate

$$
N(v)=\alpha \gamma M^{1-n} x_{1}^{1+\alpha} x^{-\alpha-2} v^{2 n-1} I_{1}+\gamma x^{-1} v^{n} I_{2}
$$

in $\Omega$, where

(14) $I_{1}=-n+m(1+\alpha+2 m \gamma) x^{-1} v^{m-n}+m \alpha(m \gamma-1) M^{1-n} x_{1}^{1+\alpha} x^{-\alpha-2} v^{m-1}$

and

$$
I_{2}=-n+C^{1-n} / T+m(1+m \gamma) x^{-1} v^{m-n} .
$$

Observing that

$$
M \geq v(x, t) \geq M\left(x_{1} / x\right)^{\gamma(1+\alpha)} \quad \text { for all }(x, t) \in \Omega,
$$

we can estimate

$$
v^{m-1} \leq M^{m-1} \quad \text { if } m \geq 1
$$

and

$$
v^{m-1} \leq M^{m-1}\left(x_{1} / x\right)^{\gamma(1+\alpha)(m-1)} \quad \text { if } m<1
$$

in $\Omega$. Subsequently, substituting these expressions in (14) and then using the datum that $x \geq x_{1}$ in $\Omega$, we deduce

$$
I_{1} \leq-n+m(1+\alpha+2 m \gamma+\alpha \mid m \gamma-1) M^{m-n} x_{1}^{-1} \quad \text { in } \Omega .
$$


Analogously, we may also derive

$$
I_{2} \leq-n+C^{1-n} / T+m(1+m \gamma) M^{m-n} x_{1}^{-1} \quad \text { in } \Omega .
$$

Since though $T>C^{1-n} / n$, it follows from (15) and (16) that we can choose $x_{1}$ so large that $I_{1} \leq 0$ and $I_{2} \leq 0$ in $\Omega$. Whence, we have shown that we can indeed select an $x_{1} \geq x_{0}$ such that $v$ is a classical supersolution of equation (1) in $\Omega$.

Armed with Lemmas 1 and 2 and with Theorem 2, we can easily prove Theorem 1. By Lemma 1,

$$
\zeta^{+}(t)=\infty \quad \text { for all } t \in\left[0, C^{1-n} / n\right) .
$$

On the other hand, by Lemma 2,

$$
u(x, T)=\mathrm{O}\left(x^{-(1+\alpha) /(1-n)}\right) \quad \text { as } x \rightarrow \infty
$$

for any $T>C^{1-n} / n$ and $\alpha>0$. But then according to Theorem 2 , there exists a $\Delta>0$ such that

$$
\zeta^{+}(t)<\infty \quad \text { for all } t \in(T, T+\Delta] .
$$

In turn this implies that

$$
\zeta^{+}(t)<\infty \quad \text { for all } t \in(T, \infty)
$$

[8]. So, since $T>C^{1-n} / n$ was arbitrary,

$$
\zeta^{+}(t)<\infty \quad \text { for all } t \in\left(C^{1-n} / n, \infty\right) .
$$

\section{THE PROOF OF THEOREM 2}

The proof of Theorem 2 is similar in spirit to the proof of Lemma 2 .

Pick $A>C$ and $\nu>\mu$. Let

$$
\delta=1 / \sigma=(1-n) \gamma-1
$$

and consider the function $v(x, t)$ defined in the closure of the domain

$$
D=(\eta, \infty) \times(0, T]
$$

where $x_{0} \leq \eta<\infty$ and $0<T<\infty$ by

$$
v(x, t)=A x^{-\gamma}\left[1-A^{n-1} \nu^{-\delta} x^{\delta} t\right]_{+}^{1 / \omega}
$$

Here

$$
[z]_{+}=\max \{0, z\} .
$$

With $N$ given by (12), we compute

$$
N(v)=x^{-1} v^{n} I_{1}+\delta \omega^{-1} \nu^{-\delta} A^{n-1+\omega} x^{\delta-\gamma \omega-1} v^{n-\omega} t I_{2}
$$


with

$$
I_{1}=-n \gamma+\omega^{-1} \nu^{-\delta} A^{n-1+\omega} x^{\delta-\gamma \omega+1} v^{1-n-\omega}+m \gamma(m \gamma+1) x^{-1} v^{m-n}
$$

and

$I_{2}=-n+m(2 m \gamma-\delta+1) x^{-1} v^{m-n}+m \delta\left(m \omega^{-1}-1\right) \nu^{-\delta} A^{n-1+\omega} x^{\delta-\gamma \omega-1} v^{m-n-\omega} t$ at all points $(x, t) \in \Omega$ where

$$
\Omega=\{(x, t) \in D: v(x, t)>0\} .
$$

Observing that $v(x, t) \leq A x^{-\gamma}$ and $t \leq \nu^{\delta} A^{1-n} x^{-\delta}$ for all $(x, t) \in \Omega$, we can estimate

$$
I_{1} \leq-n \gamma+\omega^{-1} \nu^{-\delta}+m \gamma(m \gamma+1) A^{m-n} \eta^{-(m-n) \gamma-1}
$$

and

$$
I_{2} \leq-n+m\left\{|2 m \gamma-\delta+1|+\delta\left(m \omega^{-1}-1\right)\right\} A^{m-n} \eta^{-(m-n) \gamma-1}
$$

in $\Omega$. Hence, since $\omega^{-1} \nu^{-\delta}<n \gamma$, we can always choose an $\eta$ so large that

$$
I_{1} \leq 0 \text { and } I_{2} \leq 0
$$

in $\Omega$. The function $v$ is consequently a classical supersolution of equation (1) at all points $(x, t) \in \Omega$. Noting though that $v^{m}$ is continuously differentiable in $D$, it can be verified that $v$ is subsequently a generalized supersolution of (1) in $D$.

With $\eta$ fixed, we let $T$ be so small that

$$
v(\eta, t)>u(\eta, t) \quad \text { for all } t \in[0, T] .
$$

From here it follows that

$$
v(x, t) \geq u(x, t) \quad \text { for all }(x, t) \in \bar{D} \backslash D .
$$

Thus, by the comparison principle,

$$
v(x, t) \geq u(x, t) \quad \text { for all }(x, t) \in \bar{D} \text {. }
$$

However, this means that

$$
\zeta^{+}(t) \leq \nu A^{(1-n) / \delta} t^{-1 / \delta} \quad \text { for all } t \in(0, T] .
$$

Since $\nu>\mu$ and $A>C$ were arbitrary, this validates the theorem.

\section{ACKNOWLEDGMENT}

The second author would like to thank Professor D. G. Aronson for a number of stimulating discussions concerning this work. 


\section{REFERENCES}

1. D. G. Aronson, The porous medium equation, Nonlinear Diffusion Problems (A. Fasano and M. Primicerio, ed.), Lecture Notes in Math., vol. 1224, Springer-Verlag, Berlin, 1986, pp. 1-46.

2. P. Bénilan, Evolution equations and accretive operators, Lecture notes taken by S. Lenhart, University of Kentucky, 1981, ii +129 pp.

3. J. I. Diaz and R. Kersner, Non existence d'une des frontières libres dans une équation dégénérée en théorie de la filtration, C.R. Acad. Sci. Paris Sér. I Math. 296 (1983), 505-508.

4. __ On a nonlinear degenerate parabolic equation in infiltration or evaporation through a porous medium, J. Differential Equations 69 (1987), 368-403.

5. L. C. Evans and B. F. Knerr, Instantaneous shrinking of the support of nonnegative solutions to certain nonlinear parabolic equations and variational inequalities, Illinois J. Math. 23 (1979), 153-166.

6. G. Francsics, On the porous medium equations with lower order singular nonlinear terms, Acta Math. Hungar. 45 (1985), 425-436.

7. B. H. Gilding, The occurrence of interfaces in nonlinear diffusion-advection processes, Arch. Rational Mech. Anal. 100 (1988), 243-263.

8. __ Improved theory for a nonlinear degenerate parabolic equation, Ann. Scuola Norm. Sup. Pisa Cl. Sci. (4) (to appear). Previously appearing in provisional form as: Twente University of Technology Department of Applied Mathematics Memorandum 587 (1986), ii $+42 \mathrm{pp}$.

9. R. E. Grundy, Asymptotic solution of a model non-linear convection diffusion equation, IMA J. Appl. Math. 31 (1983), 121-137.

10. A. S. Kalashnikov, The nature of the propagation of perturbations in processes that can be described by quasilinear degenerate parabolic equations (in Russian), Trudy Sem. Petrovsk. 1 (1975), 135-144.

11. - On the dependence of properties of solutions of parabolic equations in unbounded domains on the behavior of the coefficients at infinity, Math. USSR-Sb. 53 (1986), 399-410. Translation of Mat. Sb. (N.S.) 125 (1984), 398-409.

12. __ Some problems of the qualitative theory of non-linear degenerate second-order parabolic equations, Russian Math. Surveys 42 (1987), 169-222. Translation of Uspekhi Mat. Nauk 42 (1987), 135-176.

13. R. Kershner, Localization conditions for thermal perturbations in a semibounded moving medium with absorption, Moscow Univ. Math. Bull. 31 (1976), 90-95. Translation of Vestnik Moskov. Univ. Ser. I Mat. Mekh. 31 (1976), 52-58.

14. P. Rosenau and S. Kamin, Thermal waves in an absorbing and convecting medium, Phys. D 8 (1983), 273-283.

15. B. Song, The existence uniqueness and properties of the solutions of a degenerate parabolic equation with diffusion-advection-absorption, Tsinghua University Department of Applied Mathematics Science Report 88005 (1988), ii +36 pp.

Faculty of Applied Mathematics, University of Twente, Enschede, The Netherlands

Computer and automation Institute, Hungarian Academy of Sciences, Budapest, HUNGARY 